\title{
LETTER
}

\section{Plasma antioxidant capacity in critical polytraumatized patients?: methods, severity, and anatomic location}

\author{
Luis Serviá1, Javier Trujillano ${ }^{1 *}$, José Carlos Enrique Serrano², Reinald Pamplona², Mariona Badia ${ }^{1}$, Mariona Jové2, \\ Margarida Justes ${ }^{1}$, Joana Domingo ${ }^{1}$ and Manuel Portero-Otin ${ }^{2^{*}}$
}

\section{Findings}

Oxidative stress (OS) has been invoked as a relevant factor in the evolution and outcome of critical care patients. Indeed, antioxidant therapies have been used in critical care patients [1] but with controversial results [2]. This may be explained by assuming OS as a homeostatically regulated parameter and both its excess and its deficit influencing severity progression. Nonetheless, antioxidant agents could mask an OS signaling role, blocking otherwise physiological responses aimed at recovery of homeostasis [3]. We have evaluated plasma total antioxidant capacity (TAC) in traumatized patients in an ICU, and we determined its potential relationship with severity and trauma location. In a prospective observational study of ICU polytraumatized patients $(\mathrm{n}=73$, mean Acute Physiology and Chronic Health Evaluation II (APACHE II) score of $11 \pm 6$ ) of the Hospital Arnau de Vilanova (Lleida, Spain), we measured (in the first 48 hours) plasma TAC by two different methods: the ferric reducing activity/antioxidant power (FRAP) and the capacity for neutralization of the free radical 2,2'-azinobis (3-ethylbenzothiazoline)-6-sulphonic acid (ABTS) as previously described [4]. For control subjects, we used age- and gender-matched volunteers $(n=102)$. We also evaluated the contribution of antioxidant molecules (uric acid, bilirubin, and albumin) to these values. The protocol was approved by the institutional ethics committee of the Arnau de Vilanova Hospital and followed Declaration of Helsinki guidelines for studies with human individuals. All participants (or their legal representatives) gave their consent for the study.

\footnotetext{
* Correspondence: jtruji@cmb.udl.cat; manuel.portero@mex.udl.cat 'Department of Critical Care Unit, 6th floor, University Hospital Arnau de Vilanova, Rovira Roure 80, 25198 Lleida, Spain

${ }^{2}$ NUTREN-Nutrigenomics, Biomedical Research Institute (IRB) of Lleida-UdL, Biomedicine I Building, Office 3.13, Rovira Roure 80, 25198 Lleida, Spain
}

\section{Results}

As shown in Figure 1, polytraumatized patients show differences in TAC with reference to control subjects, but these differences are dependent on the technique used. Thus, ICU polytraumatized patients show higher FRAP values but lower ABTS capacity. Notably, APACHE II score influenced FRAP values (Table 1). Indeed, we found that FRAP values were inversely

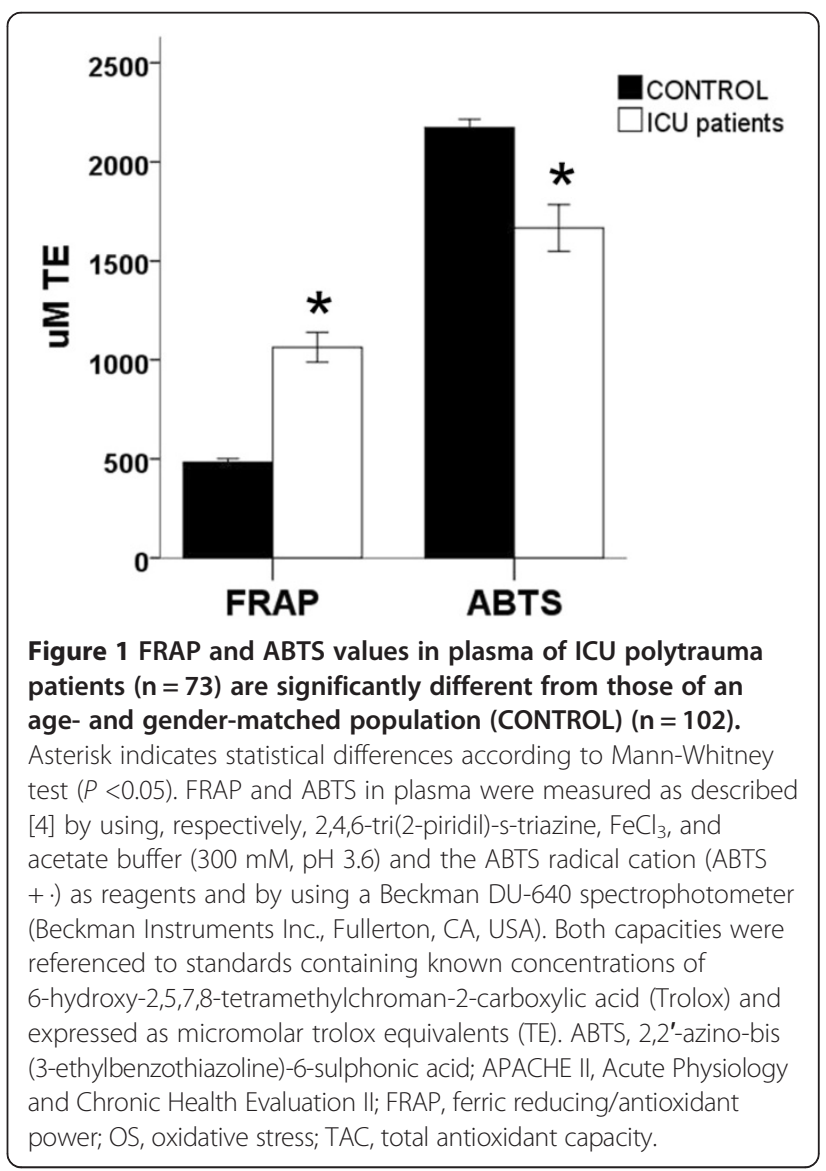

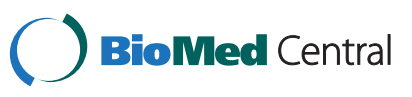

(c) 2014 Servià et al.; licensee BioMed Central Ltd. This is an Open Access article distributed under the terms of the Creative Commons Attribution License (http://creativecommons.org/licenses/by/4.0), which permits unrestricted use, distribution, and reproduction in any medium, provided the original work is properly credited. The Creative Commons Public Domain Dedication waiver (http://creativecommons.org/publicdomain/zero/1.0/) applies to the data made available in this article, unless otherwise stated. 
Table 1 Study population characteristics of ICU polytraumatized patients

\begin{tabular}{|c|c|c|c|c|}
\hline \multirow{2}{*}{$\begin{array}{l}\text { Study population } \\
\text { characteristics }\end{array}$} & \multicolumn{3}{|c|}{ APACHE II score } & \multirow[t]{2}{*}{$P$ value } \\
\hline & $\leq 7(n=28)$ & $8-14(n=23)$ & $\geq 15(n=22)$ & \\
\hline Age, years $^{a}$ & $42.4 \pm 14$ & $49.9 \pm 20$ & $49.2 \pm 19$ & 0.186 \\
\hline Male gender & 78.6 & 82.6 & 81.8 & 0.761 \\
\hline \multicolumn{5}{|l|}{$\operatorname{AIS}(\geq 3)$} \\
\hline Head & 17.9 & 43.5 & 54.5 & 0.045 \\
\hline Chest & 57.1 & 65.2 & 40.9 & 0.297 \\
\hline Abdomen & 10.7 & 8.7 & 18.2 & 0.503 \\
\hline FRAP, $\mu M T^{\mathrm{b}} \mathrm{E}^{\mathrm{b}}$ & $1,093(1,013-1,286)$ & $1,072(856-1,222)$ & $825(649-1,250)$ & 0.044 \\
\hline ABTS, $\mu \mathrm{M}$ TE & $1,763(1,555-2,035)$ & $1,765(1,054-2,008)$ & $1,825(1,405-1,982)$ & 0.852 \\
\hline Uric acid, mg/dL ${ }^{\mathrm{b}}$ & $3.8(2.0-5.9)$ & $2.5(1.5-4.6)$ & $2.1(1.2-4.6)$ & 0.056 \\
\hline Bilirrubin, $\mathrm{mg} / \mathrm{dL}^{\mathrm{b}}$ & $0.6(0.4-0.9)$ & $0.8(0.5-1.4)$ & $0.6(0.5-1.2)$ & 0.104 \\
\hline Albumin, mg/dL ${ }^{b}$ & $3.6(3.1-3.7)$ & $3.3(2.7-3.6)$ & $3.0(2.6-3.4)$ & 0.016 \\
\hline ICU mortality & 0.0 & 0.0 & 13.6 & 0.027 \\
\hline
\end{tabular}

Severity was staged according to Acute Physiology and Chronic Health Evaluation II (APACHE II) score. Values are presented as a percentage unless indicated otherwise. ${ }^{a}$ Mean \pm standard deviation; ${ }^{b}$ median (interquartile range). $P$ according to $X^{2}$ or Kruskal-Wallis test between different APACHE $I I$ groups. ABTS, 2,2'-azino-bis (3-ethylbenzothiazoline)-6-sulphonic acid; AIS, abbreviated injury scale; FRAP, ferric reducing/antioxidant power; ICU, intensive care unit; TE, micromolar trolox equivalents.

correlated with APACHE II score $(r=-0.266, P<0.01)$ suggesting that, in trauma patients, increased antioxidant response, as measured by FRAP assay, could be a pathophysiological response to stress. Albumin and uric acid concentrations reproduced the FRAP trend with severity. Reinforcing the importance of the technique and the specificities across different antioxidant assessment methods, data for the relationship of APACHE II score with ABTS do not show a significant trend $(r=0.040$, $P=0.568$ ). These results also contrast with those obtained in other ICU patients, such as those with sepsis [5].

In the multiple linear regression, FRAP values in trauma ICU patients are independently influenced by age $(\beta=0.271, P<0.021)$, APACHE II score $(\beta=-0.356$, $P<0.002)$, and head trauma $(\beta=-0.219, P<0.045)$. These results accentuate the influence of trauma location and severity in TAC changes.

Our results not only stress the importance of the method used for TAC measurement but also show that age, status severity, and anatomical location of trauma influence TAC response in ICU patients, reinforcing the need for an adequate tailoring of treatments aimed at their recovery, such as antioxidant therapies.

\section{Abbreviations}

ABTS: 2,2' -azino-bis (3-ethylbenzothiazoline)-6-sulphonic acid; APACHE II: Acute physiology and chronic health evaluation II; FRAP: Ferric reducing/ antioxidant power; OS: Oxidative stress; TAC: Total antioxidant capacity.

\section{Acknowledgments}

This work was supported by IRBLleida biobank and RETICS BIOBANCOS RD09/0076/00059 and in part by ISCIII (FIS 011-1532) and Generalitat of Catalonia (2009-SGR735). We are indebted to the ICU nurses for their help in sample extraction.

Published: 12 Jun 2014

\section{References}

1. Berger MM, Soguel L, Shenkin A, Revelly JP, Pinget C, Baines M, Chioléro RL: Influence of early antioxidant supplements on clinical evolution and organ function in critically ill cardiac surgery, major trauma, and subarachnoid hemorrhage patients. Crit Care 2008, 12:R101.

2. Heyland D, Wischmeyer PE, Wischmeyer PE, Canadian Clinical Care Trials Group: Glutamine and antioxidants in critically ill patients. N Engl J Med 2013, 369:484-485.

3. Motoyama T, Okamoto K, Kukita I, Hamaguchi M, Kinoshita Y, Ogawa H: Possible role of increased oxidant stress in multiple organ failure after systemic inflammatory response syndrome. Crit Care Med 2003, 31:1048-1052.

4. Pulido R, Jiménez-Escrig A, Orensanz L, Saura-Calixto F, Jiménez-Escrig A: Study of plasma antioxidant status in Alzheimer's disease. Eur J Neurol 2005, 12:531-535.

5. Chuang CC, Shiesh SC, Chi CH, Tu YF, Hor LI, Shieh CC, Chen MF: Serum total antioxidant capacity reflects severity of illness in patients with severe sepsis. Crit Care 2006, 10:R36.

\section{$10.1186 /$ cc13917}

Cite this article as: Serviá et al:: Plasma antioxidant capacity in critical polytraumatized patients?: methods, severity, and anatomic location. Critical Care 2014, 18:434 